\title{
Hochschule als ,offener“" Bildungskontext für lebenslanges Lernen? Befunde und Perspektiven für die empirische (Weiter-)Bildungsforschung
}

\author{
Karin Dollhausen
}

Eingegangen: 2. September 2015 / Angenommen: 9. Oktober 2015 / Online publiziert: 2. Dezember 2015 (C) Die Autor(en) 2015. Dieser Artikel ist auf Springerlink.com mit Open Access verfügbar

Zusammenfassung Das Thema der Öffnung der Hochschulbildung und von Hochschulen als institutionalisierten und organisierten Bildungskontexten für das lebenslange Lernen erfährt seit gut zehn Jahren eine besondere Aufmerksamkeit in bidungspolitischen und bildungswissenschaftlichen Diskursen. Im Zuge dessen hat sich auf internationaler, europäischer und nationaler Ebene und im Schnittfeld von Weiterbildungsforschung und Hochschulforschung ein Forschungszusammenhang etabliert, der sich auf die Offenlegung der Rahmenbedingungen, Formen und Anforderungen der gegenwärtigen und zukünftigen Entwicklung von Hochschulen im Kontext lebenslangen Lernens konzentriert. Im vorliegenden Beitrag werden hauptsächliche thematische Zugänge und empirische Befunde dargelegt und anschließend diskutiert und Perspektiven für die weiterführende Forschung benannt.

Schlüsselwörter Empirische Bildungsforschung · Weiterbildung an Hochschulen · Nicht-traditionelle Studierende $\cdot$ Lebenslanges Lernen

\begin{abstract}
The opening up of higher education and the development of higher education insti-tutions as institutionalized and organized settings for lifelong learning has been focused in educational policy and scientific discourse for more than ten years. Consequently, research at the interface between further education and higher education research at international, European and national levels has been intensified in order to disclose framework conditions, forms and challenges regarding current and expectable development of higher education institutions within the context of lifelong learning. The article highlights main topics and empirical research result with an ensuing discussion and recommendations for further topic-related research.
\end{abstract}

Prof. Dr. K. Dollhausen $(\bowtie)$

Deutsches Institut für Erwachsenenbildung - Leibniz Zentrum für lebenslanges Lernen,

Bonn, Deutschland

E-Mail: dollhausen@die-bonn.de 
Keywords empirical educational research · university continuing education · nontraditional students $\cdot$ lifelong learning

\section{Hochschulen als Einrichtungen lebenslangen Lernens: Zugänge und empirische Befunde}

Es besteht kein Zweifel daran, dass sich im Zusammenhang mit gesellschaftlichen Entwicklungen, wie sie unter Stichworten, wie etwa „Globalisierung“, ,Wissensgesellschaft“, „Digitalisierung“, „demografischer Wandel“, „Migration“" usw. beobachtet werden, die Erwartungen an Hochschulen tiefgreifend verändert haben. Sowohl von öffentlicher, bildungspolitischer als auch von bildungswissenschaftlicher Seite sehen sich Hochschulen heute mit der Anforderung konfrontiert, ihren Beitrag und ihre Rolle im Kontext des lebenslangen Lernens zu stärken, d. h. Zugänge und Möglichkeiten zur erfolgreichen Teilnahme an hochschulischen Lern- und Bildungsprozessen zu erweitern und das hochschulische Bildungsangebot auf die Bedarfe und Lernbedürfnisse einer zunehmend diversifizierten Studierendenschaft abzustimmen. Dabei gilt es zum einen, auf die gestiegenen (Höher-)Qualifizierungs- und Kompetenzentwicklungsbedarfe der Wirtschaft infolge einer zunehmend wissensbasierten, digital unterstützten und international vernetzten Produktions- und Dienstleistungsarbeit Bezug zu nehmen. Zum anderen geht es darum, auf einen gestiegenen Bedarf der erwachsenen Bevölkerung zur lebensbegleitenden Bildung und Weiterbildung zum Zwecke der persönlichen Weiterentwicklung und Selbstverwirklichung, kulturellen Bereicherung und Bewältigung biografischer Diskontinuität zu antworten. Zugleich sind Hochschulen damit herausgefordert, ihr institutionelles Selbstverständnis sowie professionell geprägte und (lern-)kulturell eingespielte Werthaltungen, Überzeugungen und Normen insbesondere in Bezug auf die Qualität und Relevanz akademischen Wissens sowie Lehren, Lernunterstützung, Beratung und Gemeinschaftsbildung (,community education“) auf den Prüfstand zu stellen und zu revidieren.

Vor diesem Hintergrund formiert sich seit gut zehn Jahren im Schnittfeld von (Weiter-)Bildungs- und Hochschulforschung ein engagierter Diskussions- und Forschungszusammenhang. Den Ausgangs- und Bezugspunkt bildet dabei der, im internationalen und europäischen Kontext sowie auch von nationalen Regierungen forcierte übergreifende Topos der Öffnung der Hochschule für bislang in der Hochschulbildung unterrepräsentierte Zielgruppen, die mit unterschiedlichen Akzentsetzungen etwa als „erwachsene Lernende“ (Mark et al. 2003), „nicht-traditionelle“ Studierende (Schuetze und Wolter 2003) oder auch als „lebenslang Lernende“ (Slowey und Schuetze 2012b) vorgestellt werden. ${ }^{1}$ Gemeinsam ist diesen unterschiedlichen Bezeichnungen, dass sie in sich heterogene Gruppen von Studierenden ${ }^{2}$ ansprechen, die der im Hochschulbereich tradierten Leitfigur der/des Studierenden,

\footnotetext{
${ }^{1}$ Im Folgenden wird im vorliegenden Originaltext im Anschluss an die in der deutschen Diskussion weitgehend eingespielte Begriffsverwendung von ,nicht-traditionellen Studierenden“ gesprochen.

${ }^{2}$ Der Begriff der/des Studierenden wird hier im Anschluss an die Begriffsverwendung in der internationalen Diskussion generell für Lernende verwendet, die das hochschulische Bildungs- und Weiterbildungsangebot nutzen.
} 
die/der als junge/r Erwachsene/r direkt nach dem Erhalt der formalen Hochschulzugangsvoraussetzung (Abitur, Matura usw.) ein Vollzeitstudium beginnt und dieses dann bis zum Abschluss hin durchläuft, nicht entsprechen. Stattdessen werden Studierende in Betracht gezogen, die etwa

- zum Zeitpunkt der Immatrikulation ein höheres Alter (25 Jahre und älter) haben,

- beruflich qualifiziert sind, aber keine formale Zugangsvoraussetzung haben,

- ein Studium und/oder weiterbildende Hochschulprogramme (z. B. Zertifikatskurse) neben der Berufs- und/oder Familientätigkeit wahrnehmen,

- aus bislang in der Hochschulbildung unterrepräsentierten Bevölkerungsgruppen (Personen aus sozial benachteiligten und einkommensschwachen Verhältnissen, Personen mit Migrationshintergrund),

- nicht direkt nach dem Schulabschluss den Weg ins Studium finden oder ein Studium nach einer längeren Pause wieder aufnehmen oder nach ein Phase der Neuorientierung ein neues Studium beginnen,

- besondere Flexibilitätsanforderungen an die Organisation des Bildungsangebots stellen, die also ein Teilzeitstudium, Fernunterricht und/oder Lernzeiten ,neben“ bzw. außerhalb der beruflichen Arbeitszeit (z. B. am Abend, am Wochenende usw.) bevorzugen.

Mit Blick auf die behandelten Themen lassen sich in der Diskussion und Forschung zur Öffnung der Hochschulbildung bis heute vor allem drei Schwerpunkte identifizieren, den chancengerechten Hochschulzugang, die Gestaltung von Übergängen in die Hochschulbildung sowie die strategische Entwicklung von Hochschulen hin zu Einrichtungen lebenslangen Lernens.

\subsection{Chancengerechter Zugang zur Hochschulbildung}

Die Thematik eines chancengerechten Zugangs zur Hochschulbildung ist an sich in der Bildungs- und Hochschulforschung keineswegs neu, sondern lässt sich mindestens bis in die späten 1960er und beginnenden 1970er Jahre zurückverfolgen, also in die Zeit der vor allem in den westlichen Industrienationen geführten Reformdiskurse und angeregten Reformprozesse. Die damaligen, auch unter der paradigmatischen Forderung nach „Bildung als Bürgerrecht“ (Dahrendorf 1965) geführten Debatten, haben in der Folgezeit einen Anstieg staatlicher Bildungsinvestitionen und eine Bildungsexpansion befördert, die nationalen Hochschulsystemen ein enormes Wachstum beschert bzw. die Entwicklung des jeweiligen Hochschulsystems von einem vormaligen Elite- zu einem Massensystem, das im Prinzip universell zugänglich ist, (Trow 1974) befördert haben. Mit Blick auf die Tatsache, dass in vielen Ländern weltweit die Beteiligung an Hochschulbildung nach wie vor ein soziales Privileg darstellt, hat die Forderung nach der Entwicklung von Hochschulsystemen und Hochschulen, die für „die Masse“ zugänglich sind, im internationalen Kontext bis heute Aktualität. Dennoch hat sich unter der Perspektive der Förderung des lebenslangen Lernens das Verständnis darüber, wie in der Gesellschaft der Gegenwart ein chancengerechter Zugang zur Hochschulbildung aussehen und gewährleistet werden kann, verändert. Vor allem geht es heute nicht allein darum, Hochschulzugänge im Prinzip „für Alle“ bzw. egalitär zu ermöglichen und dazu allgemein geltende Zugangsbestimmungen zu 
formulieren. Vielmehr geht es darum, Zugänge zur und Beteiligungsmöglichkeiten an Hochschulbildung unter Berücksichtigung der erhöhten Eigenverantwortung von Individuen für die Gestaltung von Bildungs-, Berufs- und Beschäftigungsbiografien zu erweitern und zu flexibilisieren (Field 2000). Im weiteren Sinn geht es darum, die Gestaltung von Hochschulzugängen so anzulegen, dass Hochschulbildung als individuell wählbare Option der Bildungsbeteiligung im Kontext des lebenslangen Lernens, die allen Interessierten mit persönlicher Eignung und Studierneigung offen stehen sollte, verwirklicht werden kann.

Ein bislang immer wieder bestätigtes Ergebnis von international und europäisch vergleichenden wie auch länderspezifischen Survey-Forschungen, die sich auf internationale statistische Daten etwa der OECD und der UNESCO und/oder auf nationale Daten stützen, ist die Feststellung, dass etwa gemessen an der Zahl von Immatrikulationen und dem prozentualen Anteil von Hochschulabsolventen in der Bevölkerung, die Beteiligung an Hochschulbildung zwar insgesamt weiter gestiegen ist. Dennoch erweist sich die Hochschulbildung über Kontinente hinweg in vielen Ländern als nach wie vor sozial selektiv. So kommen etwa Osborne, Rimmer und Houston zu dem Resümee: ,[...] the groups that benefit disproportionally and those who are excluded vary, but the core issue remains the same: most systems of higher education contain systemic bias towards some groups“" (Osborne et al. 2015).

Exemplarisch betrachtet, spiegelt sich dies auch und spezifischer Weise in der Entwicklung der Beteiligung an Hochschulbildung in Deutschland wider, die in international vergleichender Perspektive mitunter sogar als „exzeptionell“ bezeichnet wird (Powell und Solga 2011). So wird etwa im Bildungsbericht für Deutschland seit 2011 eine kontinuierlich positive Entwicklung bei den unter 20-jährigen Studienanfängerinnen und -anfängern im Erststudium verzeichnet. Ihr Anteil ist vor allem zwischen 2010 und 2012 deutlich angestiegen (Autorengruppe Bildungsberichterstattung 2014, S. 126). Gleichzeitig wird jedoch eine sich öffnende Schere zwischen der Beteiligung der ,traditionellen“ Studierenden und der Gruppe der nicht-traditionellen Studierenden, die erst nach einer abgeschlossenen Berufsausbildung den Weg in die Hochschule finden (,,beruflich Qualifizierte“), beobachtet. So zeigt sich seit Jahren, dass der Anteil der beruflich Qualifizierten mit und ohne Abitur, die ein Studium in Deutschland aufnehmen, insgesamt sogar rückläufig ist. Er beträgt, nach einem leichten Anstieg der Einschreibungen im Wintersemester 2009/10 von zuvor 25 auf 27\%, im Wintersemester 2011/12 nur noch 22\% (ebd., Tab. F2-19web). Der Anteil der Einschreibungen speziell von Studienanfängerinnen und -anfängern ohne Abitur ist zwar gestiegen, liegt mit insgesamt 2,6\% im Wintersemester 2011/12 jedoch nach wie vor auf einem niedrigen Niveau (ebd., Tab. F2-21web). Überraschend erscheint diese Entwicklung vor allem vor dem Hintergrund des Beschlusses der Kultusministerkonferenz (KMK) vom 6. März 2009 über die Erleichterung des Hochschulzugangs ,für beruflich qualifizierte Bewerber ohne schulische Hochschulzugangsberechtigung" (KMK 2009) sowie einer ansonsten insgesamt positiven Entwicklung der Beteiligung der erwachsenen Bevölkerung an (non-formaler) Weiterbildung über die letzten Jahre (BMBF 2014). 


\subsection{Gestaltung von Übergängen in die Hochschulbildung}

Einen weiteren, mit dem Thema des chancengerechten Zugangs zwar verwobenen, jedoch anders akzentuierten Themenschwerpunkt bildet die Gestaltung von Übergängen in die Hochschulbildung. Den auch bildungspolitisch relevanten Hintergrund bildet dabei die Frage bzw. das Problem der institutionellen „Durchlässigkeit“ in Bildungssystemen, hier insbesondere die Durchlässigkeit zwischen beruflicher Bildung und Hochschulbildung (Europäische Kommission 2011). Dem liegt die Erkenntnis und empirische Erfahrung zugrunde, dass Bildungssysteme rein quantitativ betrachtet und gemessen etwa am Bildungsniveau oder der (Weiter-)Bildungsbeteiligung oder auch gemessen am Stand der Kompetenzentwicklung der erwachsenen Bevölkerung $^{3}$ durchaus effektiv erscheinen. Jedoch bedeutet dies nicht zugleich, dass Bildungssysteme auch in Bezug auf die Förderung lebenslangen Lernens effektiv sind. Entscheidend ist, wie insbesondere John Field (2000) herausgestellt hat, die systemische Qualität von Bildungssystemen, d. h. die Koordination ihrer Institutionen als vertikal und horizontal anschlussfähig miteinander verknüpfte Orte des Lernens vom Primarbereich bis hin zur nachberuflichen Bildung (Wiesner und Wolter 2005, S. 18).

Die Frage nach der Förderung und Entwicklung von durchlässigeren Strukturen betrifft dabei vor allem, doch nicht nur traditionell „stark“ strukturierte bzw. institutionell vergleichsweise „trennscharf“ differenzierte Bildungssysteme, wie sie vor allem in mittel- und westeuropäischen Ländern (z. B. Deutschland, Frankreich, Österreich, Schweiz) anzutreffen sind. So belegen aktuelle Untersuchungen etwa zur Entwicklung im Vereinigten Königreich (UK), dessen Bildungssystem im Bereich der Berufs- und Hochschulbildung zumindest aus kontinentaleuropäischer Sicht als vergleichsweise ,verknüpft“ beschrieben wurde und wird, dass sich gerade hier, insbesondere in Wales und Schottland, eine gegenläufige Tendenz abzuzeichnen beginnt (Ertl 2014, S. 210). Bislang dominiert in der Forschung allerdings die Frage, wie in Ländern mit stark strukturierten Bildungssystemen institutionelle Differenzierungen und damit verbundene Prägungen von Bildungs- und Berufskarrieren überwunden werden können.

In diesem Zusammenhang gerät unter anderem das deutsche Bildungssystem immer wieder als eines in den Blick, das durch eine auffallend starke institutionelle Strukturierung gekennzeichnet ist und das in dieser Verfasstheit einen kaum übersehbaren, einschränkenden Einfluss auf die Gestaltung von individuellen Bildungs- und Berufskarrieren hat. So weisen Autoren, wie etwa Wolter (2012a), Powell und Solga (2011), Broek und Hake (2012) in vergleichender Perspektive in weitgehender Übereinstimmung darauf hin, dass das deutsche Bildungssystem Bildungsentscheidungen im Lebensverlauf und mithin die Gestaltung individueller Bildungsbiografien in spezifischer Weise mitprägt. Insbesondere betrifft dies die tradierte Konstellation von Berufsbildung und Hochschulbildung. Baethge (2006) hat in diesem Zusammenhang bereits vor Jahren kritisch auf das „Schisma“ im deutschen Bildungssystem

\footnotetext{
${ }^{3}$ Informationen und Daten hierzu finden sich beispielsweise in der regelmäßigen Berichterstattung der OECD, so etwa in der Reihe „Education at a Glance“ (OECD 2014), im „Skills Outlook“ (OECD 2013), besser bekannt unter dem Kürzel PIAAC, sowie im von der Europäischen Union unterstützten Adult Education Survey (AES).
} 
hingewiesen, d. h. auf die strukturell forcierte Differenzierung zwischen beruflich und akademisch orientierten Bildungs- und Berufswegen. Minks (2011) wie auch Wolter (2012a) weisen ähnlich auf die „kanalisierende“ Wirkung des Schulsystems hin. Demnach werden bereits nach Abschluss der Grundschule mit der Entscheidung für einen weiterführenden Schultyp (Hauptschule, Realschule, Fachgymnasium, Gymnasium) im Leben des Einzelnen wesentliche Weichen für die spätere Bildungsund Berufsbiografie gestellt. So resümiert Wolter (ebd., S. 45, Herv. i. O.): „Those school leavers who do not transfer to the upper level of the Gymnasium - either to its general or vocational form - normally move on to institutions of vocational training. [...] Among those who finish their school career with the Abitur, a minority continue to vocational education [...]." Konvergierend dazu betonen auch Powell und Solga (2011) die in Deutschland prägnant ausgeprägte Segregation der Domänen der Berufsbildung einerseits und der akademischen Ausbildung an Hochschulen andererseits, die trotz prinzipiell bestehender Übergangsmöglichkeiten bis heute in den tatsächlichen individuellen Bildungs- und Berufsbiographien eine pfadbestimmende Wirkung entfaltet: „Generally speaking, all these tracks ultimatively channel young people into specific positions in the labour market. The permeability between the tracks is relatively low, especially for upward mobility“ (ebd., S. 55).

Es ist daher wenig überraschend, dass in Deutschland - wie ähnlich auch in Österreich und in der Schweiz (Graf 2013) - in den vergangenen Jahren die Forschungsund Entwicklungsaktivitäten zur Frage der Gestaltung von Übergängen zwischen Berufsbildung und Hochschulbildung deutlich intensiviert worden sind. Darüber hinaus haben empirische Veränderungen in der Konstellation von Berufs- und Hochschulbildung etwa im Zuge der Implementierung von konsekutiven Studiengängen im Rahmen des Bologna-Prozesses und der Ausweitung von weiterbildenden Studiengängen („Weiterbildungsmaster“) das Forschungs- und Entwicklungsinteresse verstärkt. Bislang konzentriert sich die Aufmerksamkeit auf folgende Entwicklungen,

- die Anerkennung und Anrechnung von außerhochschulisch bzw. beruflich erworbenen Kompetenzen auf das Hochschulstudium (Freitag et al. 2011; Hanak und Sturm 2015),

- den Ausbau von transitorisch angelegten Bildungs- und Beratungsangeboten, die auf die Erleichterung von Übergängen beruflich Qualifizierter in die Hochschulbildung zielen (Freitag et al. 2015, insb. Kap. 1),

- die Kooperation zwischen Arbeitgebern und Hochschulen bei der Entwicklung und Umsetzung von weiterbildenden Studienprogrammen (Zastrow 2012; Zink 2013; Maschwitz 2014),

- die Ausweitung von dualen Studiengängen und darauf spezialisierten Hochschulen, die eine berufliche Qualifizierung mit einem Hochschulstudium kombinieren (Bundesvereinigung der Deutschen Arbeitgeberverbände und Stifterverband für die Deutsche Wissenschaft 2011; Graf 2013).

Sieht man einmal von Ergebnisdarstellungen von Modellprojekten sowie überwiegend deskriptiv angelegten Fallstudien etwa zur Entwicklung von transitorisch angelegten Bildungs-und Beratungsangeboten ab, dann zeigt sich beim gegenwärtigen Stand der empirischen Analyse, dass vor allem solche Entwicklungen, die auf eine engere kooperative Verzahnung des Hochschulkontextes (und seiner Akteure) mit 
der Berufswelt (und ihren Akteuren) zielen oder eine solche erfordern, bis dato noch deutlich problembehaftet sind bzw. ein hohes Konfliktpotenzial bergen, das sich auch, aber keineswegs nur aus sachlichen (z. B. ressourcenbedingten) und strukturellen (z. B. administrativen, zeitlichen) Inkompatibilitäten etwa zwischen Wirtschaftsunternehmen und Hochschulen heraus erklären lässt. Ein wiederkehrender Befund ist der, dass auf Seiten der Hochschulakteure vielfach noch deutliche, institutionell geprägte Vorbehalte in Bezug auf ein stärkeres kooperatives Engagement im Hinblick auf die Gestaltung von Übergängen wirksam werden. Zugleich nährt dies offenbar wiederum Vorbehalte gegenüber der Hochschule als Kooperationspartner (Maschwitz 2014, S. 36 f.). Nicht zuletzt verweist dies auf die Relevanz einer, konsequent unter der Perspektive lebenslangen Lernens geführten strategischen Entwicklung der Hochschule.

\subsection{Strategische Entwicklung von Hochschulen zu Einrichtungen lebenslangen Lernens}

Ein weiterer thematischer Schwerpunkt in der Diskussion und Forschung zur Öffnung der Hochschule für nicht-traditionelle Studierende betrifft die strategische Entwicklung von Hochschulen zu Einrichtungen lebenslangen Lernens. Die Forschung bezieht sich dabei auf einen international breit getragenen Konsens (OECD 2008; Scott 2009; Yang et al. 2015). Die umfassende Entwicklungsnotwendigkeit des Hochschulbereichs und seiner Einrichtungen wurde bereits Ende der 1990er Jahre im Bericht zur UNESCO Weltkonferenz zur Hochschulbildung als zentrales Ergebnis festgehalten: „It is now clear that, to fulfil its mission, higher education must change radically, by becoming organically flexible, and at the same time more diverse in its institutions, its structures, its curricula, and the nature and forms of its programmes and delivery systems" (UNESCO 1998, S. 2). In diesem Sinn sind auch im Rahmen des Bologna-Prozesses im Laufe der Zeit Anforderungen etwa zur Anrechnung außerhochschulisch erworbener Kompetenzen, zum Ausbau nicht-traditioneller Zugangswege zum Studium, zur Eröffnung flexibler Lernwege und Studienformen sowie zur Anpassung der Organisation und Finanzierung der Hochschulen an die Erfordernisse des lebenslangen Lernens formuliert worden (Banscherus 2010). Ebenso wurden von Seiten der professionellen Community strategische Eckpunkte für die Hochschulentwicklung formuliert, darunter etwa die unter der Federführung der European University Association (EUA) erarbeitete „European Universities” Charter on Lifelong Learning“" (EUA 2008). Und auch im bildungswissenschaftlichen Diskurs ist deutlich gemacht worden, dass bei einer konsequenten Auslegung des Konzepts des lebenslangen Lernens weitreichende Entwicklungserfordernisse etwa im Hinblick auf erweiterte Zugangsmöglichkeiten, die Aufhebung der Trennung von konsekutiven und weiterbildenden Studienangeboten, Durchlässigkeit, Flexibilität, Bedarfs- und Nachfrageorientierung, Kompetenz- und Praxisorientierung in der Lehre sowie Kooperation und Vernetzung mit externen Stakeholdern aus Wirtschaft und Zivilgesellschaft in den Blick kommen (Wolter 2010a; Remdisch und MüllerEiselt 2011; Kerres et al. 2012b).

Vor diesem Hintergrund richtet sich das Augenmerk der Forschung vor allem auf die Frage, ob und inwieweit das Konzept des lebenslangen Lernens in Hochschulen 
seit Beginn der 2000er Jahre als Leitorientierung der Hochschulentwicklung aufgegriffen wird bzw. welche Bedingungen und Faktoren eine konsequente Ausrichtung am Konzept des lebenslangen Lernens erschweren. Ein bis heute zentraler Befund aus der, zumeist auf Umfragen sowie Länder- und Fallstudien basierenden, international vergleichenden Forschung ist, dass die Entwicklung der Hochschulen bei weitem noch nicht so vorangebracht worden ist, wie es möglich und aus der Perspektive lebenslangen Lernens erforderlich wäre (Smidt und Sursock 2011; Bengtsson 2013; Dollhausen et al. 2013). So resümieren etwa Slowey und Schuetze im vergleichenden Blick auf 14 Länderstudien aus allen Kontinenten, die Ende der 1990er Jahre und dann nach 10 Jahren nochmals von jeweils nationalen Wissenschaftler/innen durchgeführt wurden (Schuetze und Slowey 2000; Slowey und Schuetze 2012a):

However, while the concept of lifelong learning featured prominently in national and international education policy discourse, the implications of this concept for higher education remained underdeveloped. Some types of institutions in the post-secondary system gradually changes to accommodate lifelong learners, but overall, higher education has been slow to adapt its mission, structures and understanding of knowledge and learning - in short its culture - to the demands for a more open, flexible and egalitarian system. Tis generally slow pace of change seems somewhat surprising in the light of calls from employers for an educated, flexible and adaptable work force, the aspirations of governments to develop 'knowledge-based economies' and 'learning societies' and, in many OECD countries, significant demographic shifts associated with ageing populations (Slowey und Schuetze 2012b, S. 4).

In Deutschland lassen sich seit einigen Jahren zwar durchaus bemerkenswerte Entwicklungen beobachten. So hat sich, wie etwa Zastrow (2012, S. 345) hervorhebt, in den vergangenen Jahren die deutsche Hochschullandschaft durchaus verändert. Die Autorin verweist in diesem Zusammenhang auf die „Liberalisierung des Bildungsmarktes“, verbunden mit ,einer imposanten Vervielfachung der Akteure im Bereich akademischer Erwachsenenbildung“, sowie auf Reformen im Sinne der Deregulierung, ,die sowohl die Steuerungs- und Verwaltungsprozesse, die Finanzierungsmodalitäten, die Personalstruktur als auch die curricularen Aspekte betreffen." Ebenso weist sie auf die Aufhebung der vormals strikten Trennung von erstem berufsbildendem Abschluss und Weiterbildung sowie auf die formale Gleichstellung von Universitäts- und Fachhochschulabschlüssen hin. Die genannten Entwicklungen beziehen sich jedoch auf formale Veränderungen, die vor allem die Wirksamkeit von veränderten Governance-Strukturen im Hochschulbereich belegen (Schimank 2007; Wolter 2012c).

Unter der Perspektive des lebenslangen Lernens tritt ein anderes Bild zutage. So zeigt sich mit Blick auf die Ausrichtung der Hochschulen, dass sich vor allem klassische Universitäten nach wie vor vielfach an einem traditionellen Bildungsauftrag orientieren, d. h. am Auftrag der Förderung der disziplinär strukturierten akademischen Ausbildung von jungen Erwachsenen, die nach dem Abitur an die Hochschule kommen, hier ein Vollzeitstudium aufnehmen und dann erst ins Berufsleben eintreten. Offenbar noch zu oft wird so übersehen, dass diese „Konstruktion des Bildungs- 
auftrags von Hochschule [...] zunehmend infrage gestellt [wird], weil sie nicht mehr der empirischen Realität der Studierenden entspricht und den weiteren demografischen Trend außer Acht lässt" (Kerres et al. 2012a, S. 9). ${ }^{4}$

Dieser veränderten Realität steht in Deutschland zudem eine nach wie vor und vor allem förder- und finanzierungspolitisch erwirkte Trennung zwischen ,grundständigen" Studienangeboten einerseits und weitgehend marktförmig zu realisierender Weiterbildung (weiterbildende Studiengänge, Angebote der wissenschaftlichen Weiterbildung) andererseits gegenüber (Fischer 2012). Entsprechend tendieren Universitäten dazu, Entwicklungsaufgaben im Kontext des lebenslangen Lernens zumeist im Bereich der wissenschaftlichen Weiterbildung zu konzentrieren, wohingegen Angebote und Organisationsformen im Bereich der grundständigen Lehre fortgeschrieben werden. Ein typisches Folgeproblem dieser Strategien besteht darin, dass sich in Universitäten neben der „klassischen“ Hochschulbildung ein Bereich der marktbezogenen Weiterbildung entwickelt, dessen Verankerung im institutionellen und organisatorischen Universitätskontext wenn nicht fragwürdig, so doch wenig gesichert erscheint (Bardachzi 2010; Wilkesmann 2012).

Zugleich wird damit ein grundlegendes Entwicklungsproblem speziell von klassischen Universitäten angezeigt. Zwar sind, wie in den meisten europäischen Ländern, die Governance-Strukturen auch im deutschen Hochschulsystem verändert worden, um Hochschulen in die Lage zu versetzen „,besser und flexibler auf gesellschaftliche und ökonomische Bedarfslagen in den entstehenden Wissensgesellschaften zu reagieren“ (Kehm 2012, S. 17). Im Kern wurde und wird dabei die „Organisationswerdung“ (ebd.) der Universität anvisiert, d. h. die Abkehr von traditionellen Formen der kollegialen akademischen Selbstverwaltung (Stichweh 2005) und die Hinwendung zur organisatorisch-rationalen, hierarchischen und arbeitsteiligen Koordination von hochschulinternen Prozessen sowie von Beziehungen zwischen Universitäten und Organisationen in der relevanten Umwelt (z. B. aus Wirtschaft, Zivilgesellschaft). Faktisch sind die Formen der Steuerung im Hochschulbereich vielfältiger geworden. ${ }^{5}$ Zugleich hat dies dazu geführt, dass sich in Universitäten, bis heute kein bestimmtes Organisationsmodell durchgesetzt hat. Vielmehr „weisen Universitäten in der Regel sowohl Merkmale von Institutionen als auch Merkmale von Organisationen auf. Als Institutionen sind sie gesellschaftliche Einrichtungen, die Wissen generieren und vermitteln; als Organisationen sind sie eine Einheit aus Lehrenden,

\footnotetext{
${ }^{4}$ Diese Kritik bezieht sich auf empirische Befunde, die zeigen, dass bereits heute auch Vollzeitstudierende vielfach neben dem Studium einer Beschäftigung nachgehen, beruflich tätig sind und/oder z. B. als Alleinerziehende neben dem Studium familiär eingebunden sind (Maschwitz und Vajna 2011). Entsprechend haben sich die Erwartungen auch der ,traditionellen“ Studierenden an die Strukturen und Organisation von Studienangeboten in eine Richtung entwickelt, wie sie im bildungswissenschaftlichen Diskurs vor allem der Gruppe der nicht-traditionellen Studierenden zugeschrieben werden. Erwartet werden etwa eine größere zeitliche Flexibilität im Rahmen des Studiums und bei Prüfungen, mehr Möglichkeiten zum Selbststudium, einen stärkeren Einbezug von Praxiswissen, die Anerkennung früherer Leistungen und eine verbesserte Beratung (Wilkesmann et al. 2011, S. 293).

${ }^{5}$ Schimank (2007) etwa, identifiziert unterschiedliche Governance-Typen, die einen Einfluss auf die Hochschulentwicklung haben, darunter die staatliche Regulation, die Außensteuerung durch den Staat oder durch andere Akteure, an die der Staat Steuerungsbefugnisse delegiert (z. B. Akkreditierungsagenturen), die akademische Selbstorganisation der Hochschule, die hierarchische Selbststeuerung der Hochschulen und der Wettbewerb in und zwischen Hochschulen.
} 
Forschenden, Lernenden, Verwaltungs- und Leitungspersonal, die arbeitsteilig miteinander kooperieren“ (Kehm 2012, S. 18). Typisch begünstigt dies in der Praxis wiederum, so Kehm (ebd., S. 23), die lediglich ,oberflächliche Anpassung an das erwartete Organisationsverhalten, während auf der Mikroebene weiterhin die traditionellen Normen und Werte Bestand haben und das Handeln der Akteure bestimmen $[\ldots]$. .

Diese spezifische Konstellation erklärt zumindest teilweise, warum es in vielen Universitäten zwar möglich ist, unter der Semantik des lebenslangen Lernens kurzfristig Maßnahmen und Aktivitäten im Bereich der wissenschaftlichen Weiterbildung auf den Weg zu bringen. Offenbar ist es aber schwer, eine von den Hochschulakteuren gemeinsam getragene und verantwortete strategische Ausrichtung auf lebenslanges Lernen sowie eine Umsetzung von damit einhergehenden Veränderungsmaßnahmen zu erwirken. Dafür, dass dies nicht nur Hochschulen in Deutschland betrifft, sprechen ähnliche Ergebnisse aus der europäisch vergleichenden Forschung (de Viron und Davies 2015).

\section{Diskussion und weiterführende Forschungsbedarfe}

Im vorangegangenen Abschnitt wurde ein - zugegeben selektiv angelegter - kursorischer Überblick über den heuten Stand der Forschung und Erkenntnis zur Entwicklung von Hochschulen hin zu Einrichtungen des lebenslangen Lernens gegeben. Stellt man vor diesem Hintergrund nochmals die im Titel des Beitrags angedeutete Frage, ob die Hochschule als ein „offener“ Bildungskontext für lebenslanges Lernen begriffen werden kann, dann drängt sich die paradoxe Antwort: „Ja und nein.“ auf. Es kommt offenbar auf die Perspektive und referenzielle Verortung der/des jeweils Antwort Gebenden an.

So besteht heute kein Zweifel mehr daran, dass sich die Öffnung von Hochschulen für lebenslanges Lernen seit Beginn der 2000er Jahre vor allem im bildungspolitischen Zusammenhang als vordringlich zu verfolgende Entwicklungsperspektive durchgesetzt hat. Zumindest mit Blick auf ihre Bildungsfunktion lassen sich Hochschulen in Anbetracht der gegebenen gesellschaftlichen Entwicklungsdynamik und damit einhergehenden Anforderungen an das permanente Lernen, die Weiterbildung und Höherqualifizierung kaum mehr anders denken. Wie gezeigt, ist damit zugleich das politische wie auch wissenschaftliche Anspruchsniveau in Bezug auf die Entwicklung von Hochschulen gestiegen bzw. in Richtung auf einen letztlich fundamentalen Wandel des Angebotsverhaltens, des institutionellen Selbstverständnisses und organisatorischen Steuerung von internen Prozessen sowie externen Hochschulkooperationen gewendet worden. Darin zeigt sich nicht zuletzt auch eine gewisse „modernistische“ Haltung, die sich von der Vergangenheit deutlich distanziert. Dies zumindest lässt sich feststellen, soweit im Blick auf die unter dem Postulat der Öffnung für lebenslanges Lernen formulierten Veränderungs- und Erneuerungserfordernisse in und von Hochschulen die in ,hergebrachten“ Strukturen und Formierungen erreichte pädagogische Leistungsfähigkeit von Hochschulen eine, bislang wenig hinterfragte, tendenzielle Geringschätzung erfährt. 
Unbestritten ist aber auch, dass sich zumindest die Hochschulen, die sich auf eine - wie immer begründete - privilegierte Stellung innerhalb des Bildungssystems beziehen, bis heute gegenüber evidenten angebots-, organisations- und schließlich institutionsbezogenen Veränderungs- und Entwicklungserfordernissen tendenziell verschließen, d. h. formal gegebene Möglichkeiten zur Erweiterung und Flexibilisierung ihres Leistungsspektrums im Hinblick auf eine effektive Förderung lebenslangen Lernens kaum ausschöpfen. Es ist bis heute eine empirisch offene Frage, ob und inwieweit eine solche Tendenz zu erklären ist. Zweifellos spielen institutionell eingespielte und wissenschaftlich-professionell begründbare Werthaltungen und normative Orientierungen eine Rolle. Aber womöglich ist deren Aktualisierung auch eine intuitive oder gar bewusste Reaktion angesichts einer modernisierungs- und innovationsversessenen Zeit, die auch die Aktionszyklen der Politik mitprägt, und in der die Forderung nach notwendigen Zeit- und Freiräumen für ein deliberates (statt ad hoc-) Entscheiden der Hochschulakteure im Hinblick auf ein systemisch-evolutionär erfolgversprechendes strategisches Entwicklungsverhalten der Hochschulen mittransportiert wird. Diese Interpretation liegt zumindest nicht fern, wenn man in Rechnung stellt, dass sich die europäische Bildungspolitik gegenwärtig bereits vom Entwicklungsimperativ des lebenslangen Lernens zu verabschieden beginnt - dies zugunsten der Betonung der „sozialen Dimension“ als zukunftsweisendem Entwicklungsauftrag für Bildungsinstitutionen, speziell Hochschulen (de Viron und Davies 2015, S. 55).

Vor diesem Hintergrund zeichnet sich der Bedarf für weiterführende Forschungen $\mathrm{ab}$, die auf die Vertiefung und Erweiterung des empirischen Wissens darüber beitragen, wie sich Hochschulen und ihre Akteure heute auf eine nicht nur veränderte, sondern sich dynamisch verändernde Anforderungssituation einstellen (können). Gefragt sind damit Forschungen, die zum einen auf ein genaueres Verstehen hochschulischer bzw. akademischer Bildung im Kontext lebenslangen Lernens abzielen und die zum anderen zur Offenlegung von Ansatzpunkten beitragen, die auf den hochschulspezifischen Wissens- und Orientierungsbedarf in Bezug auf das „Wie“ der Initiierung, Gestaltung und Umsetzung von Hochschulentwicklungsprojekten im Kontext des lebenslangen Lernens antworten. Dabei wird es erforderlich sein, das forschende Augenmerk auch und vor allem so anzulegen, dass reflexionsanregende Ergebnisse in Bezug auf im Hochschulkontext eingespielte Sichtweisen und Konstruktionen ermöglicht werde - so etwa über nicht-traditionelle Studierende bzw. beruflich Qualifizierte als Adressaten und Nutzer hochschulischer Bildungsangebote, die Rolle von Lehrenden und das Verhältnis von Lehrenden und Lernenden, den Umgang mit nicht-akademisch generiertem Wissen in der Hochschulbildung, die Bedeutung von international angelegten Vergleichsoperationen zu Verfahrensfragen, die Bedeutung von im sozialpädagogischen Bereich entwickelten Ansätzen der Lernunterstützung.

Open Access Dieser Artikel wird unter der Creative Commons Namensnennung 4.0 International Lizenz (http://creativecommons.org/licenses/by/4.0/deed.de) veröffentlicht, welche die uneingeschränkte Nutzung, Verbreitung und Wiedergabe für beliebige Zwecke erlaubt, sofern Sie den/die ursprünglichen Autor(en) und die Quelle ordnungsgemäß nennen, einen Link zur Creative Commons Lizenz beifügen und angeben, ob Änderungen vorgenommen wurden. 


\section{Literatur}

Autorengruppe Bildungsberichterstattung. (2014). Bildung in Deutschland 2014. Ein indi-katorengestützter Bericht mit einer Analyse zur Bildung von Menschen mit Behinderungen. Bielefeld: W. Bertelsmann Verlag.

Baethge, M. (2006). Das deutsche Bildungs-Schisma: welche Probleme ein vorindustrielles Bildungssystem in einer nachindustriellen Gesellschaft hat. SOFI-Mitteilungen, 34, 13-27.

Banscherus, U. (2010). Lebenslanges Lernen im Bologna-Prozess. In A. Wolter, G. Wiesner, \& C. Koepernik (Hrsg.), Der lernende Mensch in der Wissensgesellschaft. Perspektiven lebenslangen Lernens (S. 221-237). Weinheim: Juventa Verlag.

Bardachzi, C. (2010). Zwischen Hochschule und Weiterbildungsmarkt. Programmgestaltung berufsbegleitender Studiengänge. Münster: Waxmann Verlag.

Bengtsson, J. (2013). National strategies for implementing lifelong learning (LLL) - the gap between policy and reality: An international perspective. International Review of Education, 3, 343-352.

BMBF Bundesministerium für Bildung und Forschung. (2014). Weiterbildungsverhalten in Deutschland. Ergebnisse des Adult Education Survey AES Trendbericht. Bonn.

Broek, S., \& Hake, B. J. (2012). Increasing participation of adults in higher education: Factors for successful policies. International Journal of Lifelong Education, 8, 1-21.

Bundesvereinigung der deutschen Arbeitsgeberverbände \& Stifterverband für die deutsche Wissenschaft. (Hrsg.). (2011). Erfolgsmodell duales Studium. Leitfaden für Unternehmen. Berlin: BDA.

Dahrendorf, R. (1965). Bildung ist Bürgerrecht. Plädoyer für eine aktive Bildungspolitik. Hamburg: Nannen-Verlag.

De Viron, F., \& Davies, P. (2015). From university lifelong learning to lifelong learning universities: Developing and implementing effective strategies. In J. Yang, C. Schneller, \& S. Roche (Hrsg.), The role of higher education in promoting lifelong learning (S. 40-59). Hamburg: UNESCO Institute for Lifelong Learning.

Dollhausen, K., Wolter, A., Lattke, S., Scheliga, F., Spexard, A., Geffers, J., \& Banscherus, U. (2013). Developing the adult learning sector. Lot 3: Opening higher education to adults. Contract EAC 2012-0074. Final report. Brüssel: PublicationsOffice of the European union. doi:10.2766/65143.

Ertl, H. (2014). A world of difference? Research on higher and vocational education ind Germany and England. München: Herbert Utz Verlag.

EUA European University Association. (2008). European universities charter on lifelong learning. Brüssel: EUA.

Europäische Kommission. (2011). Mitteilung der Kommission an das Europäische Parlament, den Rat, den Europäischen Wirtschafts- und Sozialausschuss, den Ausschuss der Regionen. Wachstum und Beschäftigung unterstützen - eine Agenda für die Modernisierung von Europas Hochschulsystemen. Brüssel: KOM. (567 endgültig).

Field, J. (2000). Lifelong learning and the new educational order. Stoke on Trent: Trendham Books.

Fischer, H. (2012). Rechtliche Gleichgültigkeit - Lebenslanges Lernen im Spiegel gesetzlicher Vorgaben. In M. Kerres, A. Hanft, U. Wilkesmann, \& K. Wolff-Bendik (Hrsg.), Studium 2020. Positionen und Perspektiven zum lebenslangen Lernen an Hochschulen (S. 13-20). Münster: Waxmann Verlag.

Freitag, W. K., Hartmann, E. A., Loroff, C., Stamm-Riemer, I., Völk, D., \& Buhr, R. (Hrsg.). (2011). Gestaltungsfeld Anrechnung. Hochschulische und berufliche Bildung im Wandel. Münster: Waxmann Verlag.

Freitag, W. K., Buhr, K., Danzeglocke, E.-M., Schröder \&, S., \& Völk, D. (Hrsg.). (2015). Übergänge gestalten. Durchlässigkeit zwischen beruflicher und hochschulischer Bildung erhöhen. Münster: Waxmann Verlag.

Graf, L. (2013). The hybridization of vocational training and higher education in Austria, Germany, and Switzerland. Opladen: Budrich UniPress.

Hanak, H., \& Sturm, N. (2015). Außerhochschulisch erworbene Kompetenzen anrechnen. Praxisanalyse und Implementierungsempfehlungen. Wiesbaden: Springer VS.

Kehm, B. (2012). Hochschulen als besondere und unvollständige Organisationen? Neue Theorien zur, Organisation Hochschule. In U. Wilkesmann \& C. J. Schmid (Hrsg.), Hochschule als Organisation (S. 17-26). Wiesbaden: Springer VS.

Kerres, M., Schmidt, A., \& Wolff-Bendik, K. (2012a). Lebenslanges Lernen an Hochschulen - eine Einleitung. In M. Kerres, A. Hanft, U. Wilkesmann, \& K. Wolff-Bendik (Hrsg.), Studi-um 2020. Positionen und Perspektiven zum lebenslangen Lernen an Hochschulen (S. 9-12). Münster: Waxmann Verlag. 
Kerres, M., Hanft, A., \& Wilkesmann, U. (2012b). Implikationen einer konsequentn Öffnung der Hochschule für lebenslanges Lernen - eine Schlussbetrachtung. In M. Kerres, A. Hanft, U. Wilkesmann, $\&$ K. Wolff-Bendik (Hrsg.), Studium 2020. Positionen und Perspektiven zum lebenslangen Lernen an Hochschulen (S. 285-290). Münster: Waxmann Verlag.

KMK, Kultusministerkonferenz. (2009). Hochschulzugang für beruflich qualifizierte Bewerber ohne schulische Hochschulzugangsberechtigung. Beschluss der Kultusministerkonferenz vom 03.06.2009.

Mark, R., Pouget, M., \& Thomas, E. (Hrsg.). (2003). Adults in higher education. Learning from experience in the new Europe. Oxford: Verlag Peter Lang.

Maschwitz, A. (2014). Universitäten unternehmen kooperationen. Kooperationen zwischen öffentlichen Universitäten und Wirtschaftsunternehmen im Bereich weiterbildender berufsbegleitender Studiengänge. Münster: Verlag MV-Wissenschaft.

Maschwitz, A., \& Vajna, C. (2011). Berufstätige Studierende - studierende Berufstätige. Veränderte Studierendenpräferenzen und Öffnung der Hochschulen für neue Zielgruppen. In A. Strauß, M. Häusler, $\&$ T. Hecht (Hrsg.). Hochschulen im Kontext lebenslangen Lernens. Konzepte, Modelle, Realität (S. 272-281). Hamburg: DGWF. (DGWF Beiträge (50)).

Minks, K.-H. (2011). Lebenslanges Lernen und Durchlässigkeit - demografische und sozioökonomische Herausforderungen. In W. K. Freitag, E. A. Hartmann, C. Loroff, I. Stamm-Riemer, D. Völk, \& R. Buhr (Hrsg.), Gestaltungsfeld Anrechnung. Hochschulische und berufliche Bildung im Wandel (S. 21-35). Münster: Waxmann Verlag.

OECD. (2008). Tertiary education for the knowledge society. Paris: OECD.

OECD. (2013). OECD skills outlook 2013. First results from the survey of adult skills. Paris: OECD.

OECD. (2014). Education at a glance 2014 highlights. Paris: OECD.

Osborne, M., Rimmer, R., \& Houston, M. (2015). Adult access to higher education: An International overview. In J. Yang, C. Schneller, \& S. Roche (Hrsg.), The role of higher education in promoting lifelong learning (S. 17-39). Hamburg: UNESCO Institute for Lifelong Learning.

Powell, J., \& Solga, H. (2011). Why are higher education participation rates in Germany so low? Institutional barriers to higher education expansion. Journal of Education and Work, 24(1-2), 49-68. doi:10.1080/13639080.534445.

Remdisch, S., \& Müller-Eiselt, R. (2011). Öffnung, Durchlässigkeit, Vernetzung: Gemeinsam auf dem Weg zur Offenen Hochschule. Das Hochschulwesen, 1, 2-6.

Schimank, U. (2007). Die Governance-Perspektive: Analytisches Potenzial und anstehende konzeptionelle Fragen. In H. Altrichter, T. Brüsemeister, \& J. Wissenger (Hrsg.), Educational governance. Handlungskoordination und steuerung im bildungssystem (S. 231-260). Wiesbaden: VS Verlag für Sozialwissenschaften.

Schuetze, H. G., \& Slowey, M. (Hrsg.). (2000). Higher education and lifelong learners. International perspectives on chance. London: RoutledgeFalmer.

Schuetze, H. G., \& Wolter, A. (2003). Higher education, non-traditional students and life-long learning in industrialized countries. Das Hochschulwesen, 5, 183-189.

Scott, P. (2009). Access in higher education in Europe and North America: Trends and developments. Bukarest: CEPES/UNESCO.

Slowey, M., \& Schuetze, H. G. (Hrsg.). (2012a). Global Perspectives on higher education and lifelong learners. London: Routledge.

Slowey, M., \& Schuetze, H. G. (2012b). All change - no change? Lifelong learners and higher education revisited. In M. Slowey \& H. G. Schuetze (Hrsg.), Global perspectives on higher education and lifelong learners (S. 3-22). London: Routledge.

Smidt, H., \& Sursock, A. (2011). Engaging in lifelong learning: Shaping inclusive and responsive university strategies. Brüssel: EUA.

Stichweh, R. (2005). Wissen únd die Professionen in einer Organisationsgesellschaft. In T. Klatetzky \& V. Tacke (Hrsg.). Organisation und Profession (S.31-44). Wiesbaden: VS Verlag für Sozialwissenschaften.

Trow, M. (1974). Problems in the transition from elite to mass higher education. In OECD (Hrsg.), Policies for higher education. General report on the conference on future structures of post-secondary education (S. 51-101). Paris: OECD.

UNESCO. (1998). World conference on higher education. Higher education in the twenty-first century: vision and action. UNESCO Paris 5-9 october 1998 (Bd. I). Paris: UNESCO. http://www.unesco. org/new/en/education/themes/strengthening-education-systems/higher-education/reform-and-innovation/1998-world-conference/. Zugegriffen: 2. Sept. 2015. 
Wiesner, G., \& Wolter, A. (2005). Einleitung. In G. Wiesner \& A. Wolter (Hrsg.), Die lernende Gesellschaft. Lernkulturen und Kompetenzentwicklung in der Wissensgesellschaft (S. 7-44). Weinheim: Juventa Verlag.

Wilkesmann, U. (2012). Strukturelle Verankerung. Die Dilemmata des lebenslangen Lernens an Hochschulen. In M. Kerres, A. Hanft, U. Wilkesmann, \& K. Wolff-Bendik (Hrsg.), Studium 2020. Positionen und Perspektiven zum lebenslangen Lernen an Hochschulen (S. 52-58). Münster: Waxmann Verlag.

Wolter, A. (2010a). Die Hochschule als Institution lebenslangen Lernens. In A. Wolter, G. Wiesner, \& C. Koepernik (Hrsg.), Der lernende Mensch in der Wissensgesellschaft. Perspektiven lebenslangen Lernens (S. 53-79). Weinheim: Juventa Verlag.

Wolter, A. (2012a). Germany: From individual talent to institutional permeability: Changing policies for non-traditional access routes in German higher education. In M. Slowey \& H. G. Schuetze (Hrsg.), Global perspectives on higher education and lifelong learners (S. 43-59). London: Routledge.

Wolter, A. (2012b). Studium neben dem Beruf - eine Realisierungsform lebenslangen Lernens an Hochschulen. In M. Kerres, A. Hanft, U. Wilkesmann, \& K. Wolff-Bendik (Hrsg.), Studium 2020. Positionen und Perspektiven zum lebenslangen Lernen an Hochschulen (S. 271-284). Münster: Waxmann Verlag.

Wolter, A. (2012c). State, market and institution in German higher education - new governance mechanisms beyond state regulation and market dynamiks. In H. G. Schuetze, G. Alvarez Mendiola, \& D. Conrad (Hrsg.), State and market in higher education reforms. Trends, policies and experiences in comparative perspective (S. 129-147). Rotterdam: Sense Publishers.

Yang, J., Schneller, C., \& Roche, S. (Hrsg.). (2015). The role of higher education in promoting lifelong learning (S. 17-39). Hamburg: UNESCO Institute for Lifelong Learning.

Zastrow, J. (2012). Die institutionelle Neuausrichtung der wissenschaftlichen Management-weiterbildung - ein Beitrag zur Entwicklung des organisationalen Felds der wissenschaftlichen Weiterbildung in Deutschland. In U. Wilkesmann \& C. J. Schmid (Hrsg.), Hochschule als Organisation (S. 345-362). Wiesbaden: Springer VS.

Zink, F. (2013). Wissenschaftliche Weiterbildung in der Aushandlung. Die Akteuere und ihre Themen in interinstitutionellen Aushandlungsprozessen im Kontext kooperativer Angebotsentwicklung. In K. Dollhausen, T. C. Feld, \& W. Seitter (Hrsg.). Erwachsenenpädagogische Kooperations- und Netzwerkforschung (S. 133-156). Wiesbaden: Springer VS. 